Seit Jahren kenne und schätze ich Jean Martin als vielseitig interessierten Redaktionskollegen und Freund. Für die Schweizerische Ärztezeitung ist er auch insofern ein Glücksfall, als sich bei ihm humanistisches Engagement mit Schreibfreude und -talent paaren, wovon insbesondere der "Kulturteil» der SÄZ in reichem Mass profitiert. Seinem neuen Buch, das nachfolgend besprochen wird, wünsche ich viele Leserinnen und Leser - auch über den französischen Sprachraum hinaus.

Bruno Kesseli, Chefredaktor SÄZ

\title{
Des repères pour choisir
}

\section{Marc-Antoine Bornet}

Médecin assistant, Service de médecine interne, CHUV

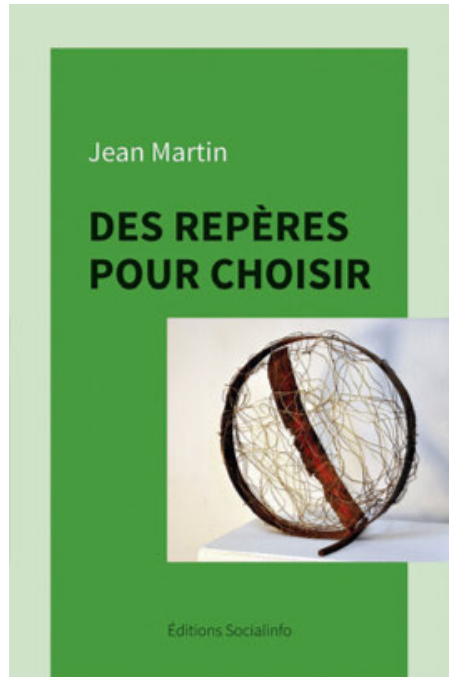

Jean Martin

Des repères pour choisir

Lausanne: Editions Socialinfo; 2017.

148 pages. $24 \mathrm{CHF}$.

ISBN 978-2-9701-0014-0

Comment s'orienter face aux multiples défis rencontrés en médecine, mais aussi dans la société? Comment faire pour bien faire? Par son ouvrage, le Dr Jean Martin nous offre de partager ses réflexions. Ethicien engagé, ancien médecin cantonal vaudois, il se définit «d'extrême-centre, au nom de l'intérêt général». Ses textes nous permettent d'explorer de nombreux thèmes actuels, reflétant notamment quelques rencontres et lectures qui l'ont touché, avec toujours en filigrane des valeurs humanistes.

\begin{abstract}
Le patient
Différentes situations et stades de vie sont évoqués, mettant en avant l'importance du dialogue et du contact humain pour soigner, mais aussi pour vivre ensemble. Le patient est reconnu dans sa globalité, incluant sa dimension existentielle et son contexte de vie. Une constante dans les textes: le respect inconditionnel de l'être humain, si fragile et si différent qu'il puisse être. Ce respect s'ancre au quotidien par une sagesse pratique pour toute prise de décision. «Chaque situation doit être considérée pour elle-même dans ses différentes dimensions, avec la personne concernée, en dialogue avec les proches signifiants.» Un exemple: comment répondre à des parents qui refuseraient les tests de dépistage à la naissance par le prélèvement d'une goutte de sang au talon du nouveau-né? «Des mesures autoritaires sont inadéquates. Alors... écouter et dialoguer.»
\end{abstract}

\section{L'éthique}

Durant son activité, le Dr Jean Martin a observé l'émergence de nombreuses questions éthiques. Néanmoins, il constate que les questions auxquelles il était soumis lors de sa formation ne sont pas encore résolues et restent d'actualité, d'une gravité croissante. «Malgré la multiplication des comités d'éthique, on traite surtout de questions partielles, en ne trouvant

\section{Comment s'orienter face aux multiples défis rencontrés en médecine, mais aussi dans la société?}

pas le temps de consacrer assez d'attention aux questions 'surplombantes' de l'évolution de la biomédecine et de la société.» L'auteur invite à une évolution de 
l'éthique: «A la dérive où nous mène la 'Petite éthique' actuelle, se substituerait le choix collectif d'options mûrement débattues. [...] Pourtant, sous peine de fin d'histoire, il faudra trouver les voies et moyens d'une ‘Grande éthique’ planétaire.»

\section{La société}

Pour le Dr Jean Martin, l'éthique devrait être au cœur de la société: elle va plus loin que la loi, par exemple dans des situations légalement possibles mais éthiquement inacceptables. "Il ne serait pas bon de vouloir faire régler en détail par l'Etat tout ce qui se passe entre le professionnel et son malade: il y a des thèmes sur lesquels on pourrait (re)passer du droit à l'éthique.» Le bon sens et le souci constant de l'autre doivent rester au premier plan: «Les professionnels doivent se montrer dignes de la confiance que, cas échéant, on leur témoigne en renonçant à légiférer. [...] Eviter d'élaborer des lois superflues demande une solide dose d'esprit et de compréhension civiques.» La société ne se limite pas à notre environnement proche. Ayant débuté sa carrière dans l'humanitaire, l'auteur sait ouvrir sa réflexion sur une société globale.
«Prendre la mesure des choses dans d'autres environnements!» Le monde se complexifie: «intéressé depuis toujours à la marche du monde, je le vis aujourd'hui comme désemparé, 'illisible'». Il souhaite avant tout

\section{Une constante dans les textes: le respect inconditionnel de l'être humain, si fragile et si différent qu'il puisse être.}

mettre en avant «la coexistence pacifique et respectueuse entre cultures, ethnies, religions et une convivialité, soulignant que la différence c'est intéressant et enrichissant».

\section{La Terre}

Ouvrant le livre au-delà de la médecine, une autre ligne forte de l'auteur est son engagement pour la protection du climat. Une forme de respect envers les générations de demain.

Un livre de partage, de proposition et d'exercice à la prise de responsabilité au quotidien. Un regard bienveillant et profond sur notre société. 